\title{
THE USE OF PHYSIOTHERAPY IN THE TREATMENT OF WOUNDS
}

\author{
By Hazel Baines, M.C.S.P., T.M.E., and Lois Dyer, M.C.S.P.
}

$T$ HE following are some of the well tested techniques used in the Physiotherapy Department of the Johanneshurg General Hospital on cases which have received the usual care, but have been slow or unsuccessful in their response to other forms of treatment.

When a patient is referred to the Physiotherapy Department, the aim is to obtain a quick response and a permanent result with maximum function. There are certain fundamental principles to be observed if these aims are to be fulfilled. The cooperation of the patient and the nursing staff must be achieved, and the interest of the physiotherapist maintained throughout. 'The quickest way to lose a patient's co-operation is to give a disinterested treatment. Wounds must be carefully observed at each attendance of the patient, since their condition can change so rapidly. Suitable apparatus must of course be available, and work must be performed under as sterile conditions as possible.

\section{Examination of Patient}

The complete history of the patient must be studied, noting particularly if there are any metabolic disturbances which may warrant modification of treatment. The cause of the wound must obviously be ascertained, and any previous treatment, such as skin grafting, should be noted. It is pointless trying to heal a would or sinus if there is any underlying bone infection, or syphilitic condition for example, so that these factors must be eliminated by $\mathrm{X}$-raying the part.

Local examination of the part must be carried out very carefully. The wound should be measured, and any sinus noted. The appearance of the wound itself should be recorded, e.g., the presence of pus, overgranulation, epithelial tissue, etc. The dressings taken off at each treatment must be examined to see if there is an increased discharge, or if pus is present when previously there was none. The skin condition surrounding the area should be inspected for pustular formation, oedema, or poor texture. The local blood supply will obviously effect the rate of healing, e.g., the blood supply of a toe is not as well established as that of the abdomen. The presence of pain is another important factor which may have to be taken into consideration.

\section{Aims of Treatment}

By inproving the circulation to the affected area, an increase of leucocytes is brought about, and oedema is dispelled. Bacteria must be destroyed and re-infection prevented. Relief of pain must be accomplished, and increased pain can be prevented by the application of comfortable dressings, and their careful removal. It is important to improve the condition of the tissues surrounding the wound and of course, to promote healing. The mobility of the joints which may be directly or indirectly affected must be maintained, and adherent scar tissue pre- vented if possible. Steps must be taken to improve the general health of the patient, and thus raise the resistance to infection.

\section{Means}

Tliere are many ways of improving the circulation, and all methods can be employed viz.: Diathermy, infra-red, radiant heat and ultra violet light. Massage, faradism, sinusoidal current, and active exercise are all effective in increasing the circulation to specific areas, but are not used as often as the first methods. Massage and faradism under pressuré are particularly useful for long standing varicose ulcers where there is brawny oedema and a.much impaired blood supply. The massage is adapted as in the Bisgaard method of treatment. It is usually not used for acute septic wounds.

The destruction of bacteria is effected by the use of the short ultra-violet rays, i.e., 2900-1849 Angstrom units, used at a distance of not more than 15 inches from the patient. The improved supply of leucocytes to the part brought about by the circulatory improvement, will naturally help to combat infection. Specific anti-biotics will have the same effect, though care must be taken that these are not administered routinely, without first ascertaining by swab and culture that they will help.

Re-infection can largely be prevented by general asepsis, particularly with regard to dressings. The patient must be instructed not to interfere with his dressings, unless he is carefully and specially instructed to do so.

Healing is promoted by the use of ultra-violet light and the application of suitable dressings. The general health can be improved by general irradiation.

Active exercise will retain mobility of joints, help, to prevent adherent scar tissue and stimulate circulation.

The relief of pain is effected by improving the blood supply, thus removing inflammatory products and reducing oedema. Special care must be taken over the removal of dressings. They should never be pulled off, for not only does this cause pain, but it is very likely that new epithelium will be pulled off at the same time. The patient should be warned that treatment will probably increase the discharge from the wound. If dressings are sticking, whicl can to a large extent be prevented by the choice of a suitable type of dressing, they can be soaked off with peroxide of hydrogen, eusol, or sterile water.

For burn cases, where large areas are involved, we have found that complete saline baths, using a normal saline solution and a temperature of 98-100 degrees Fahrenheit, give the patient a great deal of relief and comfort, in that the dressings come off easily, and the saline has a certain antiseptic value. Movements are given in the bath, while the dressings are off, so the fullest range can be obtained. 
General Considerations in the Technique of Using Short Wave Diatherny and Ultra-Violet Light.

\section{Short Wave Diathermy}

(1) Skin sensation must be tested, and at least one electrode must be placed over an area of normal sensation. This is of notable importance in the treatment of trophic ulcers of paraplegics, for example, or when treating diabetics, whose sensation is so often impaired.

(2) Freshly applied dry dressings should cóver a wound during treatment. If elastoplast is in the field, there is always the danger of a burn occurring under the plaster, due to the accumulation of sweat which cannot evaporate. Rubber drains in the tissues will have the same effect.

(3) The position of the patient is an important factor which is often ignored. The part being treated should be comfortably supported, with the patient lying, half-lying, or sitting, whichever is more comfortable and convenient for the positioning of electrodes.

(4) It is preferable to use an electric field rather than a magnetic one, so that the underlying blood supply is stimulated directly, instead of producing heat in the superficial tissues, which will only reflexly affect the deeper vessels. Because of this, condenser electrodes are used in preference to a coil, whether round the limb or in the form of a drum or pancake. In addition to this, air spacing between the electrodes and the tissues is more comfortable than having towels covering the area.

The size of the electrodes depends on the size of the area to be treated, and they should be placed in such a way that they are parallel to the skin, and concentration of heat over any prominence is avoided as far as possible.

A very convenient and effective technique is the co-planar method, when the electrodes are placed on either side of the wound, on the same surface. The inter-electrode distance should be at least $2 \frac{1}{2}$ times the skin electrode distance. By means of this method, the field is concentrated directly beneath the wound. The heat formation on the surface is minimised, thereby reducing the thermal effect on the bacteria themselves, which might cause a spread of infection. This is especially a useful technique for the treatment of boils and carbuncles, whicl would otherwise become heated at their most prominent points.

(5) All metal objects sliould be removed from the area of the field, since a concentration of current will take place on these objects and a burn may result, e.g. Metal in the tissues, such as a SmithPetersen pin, or clips. If treatment is given in a wheel chair or on a stretcher, care must be taken to see that there is sufficient dielectric between the metal portions of these and the leads and electrodes, so as to prevent loss of energy to the patient.

(6) Skin electrode distance depends on the particular machine being used, and the depth of the lesion. A minimal spacing is used when through lieating is not required. It is advisable to remove clothing in the area of the wound before treatment, so as to achieve accurate positioning of the electrodes, and to avoid any possibility of concentration of current due to sweating in the clothing.

(7) For all acute infections an athermic dosc should be given, so as not to increase congestion or stimulate bacterial activity. This is progressed to a mild thermal, and later a thermal treatment to have a more marked effect on the circulation. Treatment is given for 5-10 minutes in the early stages, and progressed to 15-20 later. Frequency of treatment depends on whether other means of stimulating the circulation are being used, such as ultra-violet light; but some form of treatment is required at least once a day.

\section{Ultra-Violet Light}

(1) A test dose should be given to the area surrounding the wound, to determine any sensitivity of the patient.

(2) The position of the patient must be considered as for any treatment, When treating a wound it is most important that it can be examined in a good light. All areas which are, not intended for irradiation should be protected to prevent any unnecessary reaction.

(3) If the area is small enough, time is saved by the use of the Kromayer lamp, otherwise any ultraviolet lamp with a good output can be used.

(4) The wound itself and the surrounds must be cleansed with sterile swabs, to remove any substance which will prevent maximum absorption. Good cleansing agents are eusol, sterile water, or spirit provided it is not used on the wound itself.

(5) Asepsis of instruments, towels, hands, applicators or any apparatus which will be in close contact with the wound, is of vital importance. The use of a mask is advocated if the physiotherapist is likely to breathe over the wound for any length of time. Applicators should be soaked in pure Dettol prior to use, washed with sterile water, and then cleansed with Dettol after the treatment.

(6) Inspection of the wound at each treatment must be carefully carried out, to consider dosage and the effect of th'e previous treatment.

(7) ! By the use of the Inverse Square Law, any alteration in the treatment distance can be calculated. A useful formula is the following:-

$$
\text { New time }=\frac{\text { Old time } \times \text { New distance (squared) }}{\text { Old distance (squared) }}
$$

So that when reducing 18 minutes @ $3 \mathrm{ft}$. to treatment@2ft.,

$$
\text { New time }=\frac{18 \times 2 \times 2}{3 \times 3}=8 \text { minutes. }
$$

To calculate erythema doses from a 1st degree:2nd degree is 21 times a 1 st degree.

3 rd degree is 5 times a lst degree.

4th degree is 10 times a 1st degree.

$2 \times 4$ th degree is 20 times a 1 st degree.

To progress dosage:-

To obtain a $1^{\circ}$ on a $1^{\circ}$ add $25 \%$ of previous dose.

To obtain a $2^{\circ}$ on a $2^{\circ}$ add $50 \%$ of previous dose.

To obtain a $3^{\circ}$ on a $3^{\circ}$ add $75 \%$ of previous dose.

A 4 th degree blister is not progressed since it is not necessary to repeat it on normal skin. It must be remembered that when treating an open area, there will be no pigmentation to act as a protection, and therefore the dose does not need to be progressed.

If an applicator is used, for every inch of the applicator, an additional dose is added to the original.

e.g. 1st degree in contact is one second.

$$
\text { A double fourth degree is } 20 \text { seconds. }
$$

With a three-inch applicator a fourth degree is 80 seconds. 
(8) The surrounds of the wound should be treated first, since when the wound itself is treated, the edges will be protected with vaseline, and this would prevent full absorption in that area. The base of the wound can be included in the general treatment, and the dosage it receives then can be taken into consideration if necessary when treating it separately. It is very rarely that the surrounds of a wound receive a dosage longer than the base, since the surrounding skin condition should be improved with a few treatments.

(9) When treating the base of a wound, the edges must be protected to prevent any destruction of new epithelium. Sterile vaseline is applied to the edges of the wound, and a piece of lint cut to its exact size placed on this.

(10) To obtain maximum absorption, rays must strike the area at $90^{\circ}$, and this may necessitate irradiation on more than one plane.

(11) It is important that the actual base of the ulcer be treated. If necessary one must probe a sinus and treat it with a Kromayer applicator. It is useless closing a wound if there is a sinus underneath it.

(12) If the area does not seem to be responding to the dosage it is being given, then the dose must be progressed until a favourable response is obtained.

(13) The choice of dressing is of prime importance, since it must be soothing and comfortable. It is preferable to use a dressing which does not contain water, since it quickly evaporates and is inclined to adhere. Adverse reactions must be watched for, since some patients are sensitive to certain types of dressing.

Useful non-adherent, soothing dressings are acraflavine emulsion, eusol and paraffin, tulle gras and penicillin cream. Some authorities state that cod liver oil used in conjunction with ultra-violet light promulgates the formation of Vitamin D. Apart from the fact that it is a rather unpleasant dressing to deal with, because of its smell, we have been very pleased with the cases for whom we have used it.

(To be continued in the October issue when the histories of several cases treated on these lines will be given.)

\section{SOUTH AFRICAN SOCIETY OF PHYSIOTHERAPISTS.}

Members of the Society receive Hospital Training.

They are qualified to administer Massage, Remedial Exercises, Electrical, Ultra-Violet Ray and Hydro-therapy treatments,

The Society was formed in 1924 and is the only body of Physiotherapists in this country to receive the full recognition and support of the South African Medical Association.

S.A.S.P. Members do not advertise individually and pledge themselves to treat patients only under medical direction. All members of the Society are eligible for enrolment on the National Register of the South African Medical and Dental Council.

Names and addresses of members practising in any district can be obtained from the General Secretary.

All communications should be addressed to:

MISS LOIS DYER, PHYSIOTHERAPY DEPARTMENT, GENERAL HOSPITAL, JOHANNESBURG.

\section{S.A. SOCIETY OF PHYSIOTHERAPISTS.}

\section{President :}

Miss J. M. D. Crosby.

Physiotherapy Department, National Hospital, Bloemfontein.

Registrar : Mr., A. Rothberg, W.N.L.A. Hospital, Eloff Street Extension, Johannesburg.

General Secretary : Miss S. Oosthuizen, 53 Pasteur Chambers, Jeppe Street, Johannesburg.

General Treasurer : Miss H. M. Benford, 47 Lister Buildings, Jeppe Street, Johannesburg.

Appointments Secretary : Mesdames Douglas, Benford and Burr, 47 Lister Buildings, Jeppe Street, Johannesburg.

Editor : Miss Lois Dyer.

Journal Treasurer: Mrs. M. Levy, 8 Shiplake Road, Auckland Park, Johannesburg.

\section{ADVERTISEMENT RATES.}

\section{General Advertisements.}

Half Front Page $£ 10 \quad 0 \quad 0$

Inside Whole Page $£ 10 \quad 10 \quad 0$

Inside Half Page

$£ 5 \quad 10 \quad 0$

Inside Quarter Page

$£ 3 \quad 5 \quad 0$

Outside Back Page

Classified Advertisements. Practices for sale, etc.)

5/- per Line-up to 10 Lines.

3/- per Line-for each additional Line or part thereof.

A discount of 10 per cent. is allowed to subscribing members of the S.A. Society of Physiotherapists.

Members are requested to support the advertisers who use these pages. Please mention this Journal when making purchases. 